This is an open access article distributed under the terms of the Creative Commons BY-NC-ND Licence

\title{
Genes involved in stress signals: the CBLs-CIPKs network in cold tolerant Solanum commersonii
}

\author{
S. ESPOSITO, V. D’AMELIA, D. CARPUTO*, and R. AVERSANO* \\ Department of Agricultural Sciences, University of Naples Federico II, Via Università 100, Portici, Italy
}

\begin{abstract}
Several studies revealed the important contribution of calcineurin B-like (CBLs) and CBL-interacting kinase (CIPKs) genes in transmitting stress signals in plants. Taking advantage from the genome sequences of the cultivated potato Solanum tuberosum and its wild relatives S. commersonii and S. chacoense, we identified for the first time 10 CBLs and 26 CIPKs genes in each species. The $C B L s$ and $C I P K s$ derived from tandem duplications indicate that these gene families in potato mainly arise through amplification mechanisms. Once annotated, we compared the par excellence model of Arabidopsis thaliana with $S$. commersonii, the potato model species for studying cold tolerance. We found that four ScCBL proteins (ScCBL1, ScCBL4a, ScCBL4b, and ScCBL9) started with a conserved N-myristoylation motif (MGXXXS/T), which might function in membrane targeting of the CBLs-CIPKs complex. Additionally, expression analyses of $S$. commersonii $C B L$ and $C I P K$ genes based on RNAseq revealed diverse expression patterns following various abiotic and biotic stresses and in the four tissues analyzed (flowers, leaf, roots, and tubers). Data also suggest that the ScCBLs-ScCIPKs complex may be more responsive to abiotic rather than biotic stimuli. Overall, the results described in the present work will be useful for future investigations and for functional characterization of individual CBLs and CIPKs in Solanum.
\end{abstract}

Additional key words: abiotic and biotic stresses, regulation of gene expression, signal transduction, wild potato.

\section{Introduction}

To adapt to various environmental stimuli, plants have evolved a series of complex signal transduction mechanisms. These pathways activate when plants sense external stimuli through plasma membrane, triggering a surge of $\mathrm{Ca}^{2+}$ into the cytosol. The up-take is perceived by calcium-binding proteins, including $\mathrm{Ca}^{2+}$-dependent kinases (CDPKs), calcineurin B-like (CBLs), and CBL interacting kinases (CIPKs). These function as sensor molecules receiving signals from cellular calcium and transmitting them to downstream pathways ( $\mathrm{Li}$ et al. 2016). Depending on the nature of the response domain (either a kinase or transcription regulation domain), $\mathrm{Ca}^{2+}$ sensor proteins are classified into sensor relays or sensor responders. Among sensor relay proteins, CBLs are thought to play a central role in the $\mathrm{Ca}^{2+}$ signaling pathway in plants and some protozoans (Tuteja et al. 2007), binding $\mathrm{Ca}^{2+}$ with their four conserved EF hand motifs (Kudla et al. 1999). Once bounded $\mathrm{Ca}^{2+}$, CBLs specifically target a group of sucrose non-fermenting 1-related serine/threonine proteins
(SNF1), namely CIPKs, to transduce the perceived calcium signal (Shi et al. 2000). Commonly, CIPK proteins consist of a conserved $\mathrm{N}$-terminal kinase domain and a $\mathrm{C}$-terminal regulatory domain, which is separated from the former by a variable junction domain (Cheng et al. 2002). In particular, $\mathrm{Ca}^{2+}$-bound-CBLs interact with and activate the catalytic domain of targeting CIPKs through conserved motifs (namely NAF or FISL). Among all the interactions between sensor relay proteins and sensor responders, the CBL-CIPK network serves as an essential signaling network regulating plant responses to low temperatures, salinity, $\mathrm{K}^{+}$deficiency, excess of $\mathrm{Mg}^{2+}$, and drought (Yang et al. 2008, Held et al. 2011, Huertas et al. 2012). It also modulates growth and development, absorption and/or transport of nitrate, ammonium, and iron, sustaining $\mathrm{H}^{+}$ homeostasis, and transduction of reactive oxygen species signals in plants (Pandey et al. 2015, Thoday-Kennedy et al. 2015, Mao et al. 2016). Therefore, the identification and comprehensive study of these sensors may provide useful insights for plant breeding.

Nowadays, the identification of genes, transcripts,

Submitted 23 January 2019, last revision 24 April 2019, accepted 6 May 2019.

Abbreviations: aa - aminoacids; CBL - calcineurin B-like; CIPK - CBL-interacting kinase; PGGD - plant genome duplication database; RPKM - reads per kilobase of transcript per million mapped reads.

Acknowledgments: This work was carried out within the project Development of potato genetic resources for sustainable agriculture (PORES) funded by the University of Naples Federico II. We thank Mr. Raffaele Garramone for his technical assistance.

* Corresponding authors; fax: (+39) 081 2532124, e-mails: raversan@unina.it; carputo@unina.it 
and proteins useful for breeding involves the use of new advanced bioinformatics tools. Among them, highthroughput genome sequencing efforts have dramatically increased knowledge of and ability to discover and characterize gene families in plants. For example, taking advantage from the genome sequence of Solanum commersonii (Aversano et al. 2015), we have disclosed and characterized several gene families (D'Amelia et al. 2014, 2018, Aversano et al. 2017, Esposito et al. 2018a,b). So far, bioinformatics has also been successfully used in the identification of both CBLs and CIPKs in different species. For example, Mohanta et al. (2015) described the CBLs gene family in 38 different plant species (among them Glycine max, Arabidopsis thaliana, Cucumis sativus, $S$. lycopersicum, and $S$. tuberosum) whereas many CIPKs genes have been described in Arabidopsis, Brachypodium distachyon, rice, and maize (Kolukisaoglu et al. 2004, Chen et al. 2011, Wang et al. 2015).

Potato (Solanum tuberosum L.) is the third most important crop in the world. It is sensitive to a wide range of biotic and abiotic stresses leading to losses in production almost every year. Its susceptibility might be due to the domestication during which the cultivated forms have lost critical genes important for adaption to the environmental challenges, as already reported in G. $\max$ (Lam et al. 2010). For these reasons, a number of wild tuber-bearing Solanum species represent valuable genetic resources to improve resistance traits (Machida-Hirano et al. 2015, Mori et al. 2015). Among wild potatoes, S. commersonii is not only the most tolerant to low temperatures, but also possesses a high capacity to cold acclimation (i.e., ability to increase cold tolerance after exposure to low, nonfreezing temperatures) (Palta and Simon, 1993) and resistances to root knot nematode, soft rot and blackleg, bacterial and verticillium wilt, Potato virus $X$, tobacco etch virus, common scab, and late blight (Hanneman and Bamberg 1986, Hawkes 1990, Micheletto et al. 2000, Carputo et al. 2009, 2013). Since its genome sequence is available (Aversano et al. 2015), it has become a model species for studying plant tolerance to cold as well as other stresses. Despite extensive studies of the $C B L-C I P K$ network in other important crops, there are no reports regarding a comprehensive and detailed analysis of these gene families in potatoes. Also, how CBL-CIPK evolved in wild tuber-bearing potato species is still unknown. In this study, we exploited the genome sequence of three potato species (the cultivated $S$. tuberosum and its wild relatives $S$. commersonii and $S$. chacoense) to identify genes belonging to both $C B L$ and $C I P K$ families. Once genes were identified, we compared our model species for studying plant tolerance traits ( $S$. commersonii) with the "par excellence" model $A$. thaliana. Further aim was to analyze $S c C B L s$ and $S c C I P K s$ gene families and their phylogenetic relationship, gene structure, protein motifs, gene duplications, and divergence.

\section{Materials and methods}

In silico identification of $C B L$ and $C I P K$ genes: Solanum commersonii, S. tuberosum, and $S$. chacoense $C B L$ and $C I P K$ genes were identified through the following steps: 1) all well-characterized Arabidopsis CBLs and CIPKs were downloaded from TAIR (https://www.arabidopsis. org/index.jsp) database and aligned through ClustalW (Larkin et al. 2007); 2) the alignment file was used to build a similarity matrix using hmmr v. 3.2.1 (hmmer.org); 3) the resulted matrix was run as a bait against the $S$. commersonii, $S$. tuberosum, and $S$. chacoense genomes with e-values $<1 \mathrm{e}^{-5}$; and 4) only genes with an e-value $<10^{-5}$ and an identity $>55 \%$ were regarded as $C B L$ and $C I P K$ genes. The identified CBLs and CIPKs were further confirmed manually checking the specific domains using the NCBI search domain online tool (Marchler-Bauer et al. 2015). Names were assigned based on their orthologs in Arabidopsis using bootstrap replicates of the maximum likelihood phylogenetic tree (values higher than 50). In particular, only one-to-one orthologs were considered when genes allocated on the same clade in the phylogenetic tree with Arabidopsis. Otherwise, the names of the CBL and CIPK were assigned using the prefix-like and/or prefixspecific.

Phylogenetic analysis of gene structure and conserved motifs: All CBL and CIPK protein sequences and those of Arabidopsis were aligned using muscle (https://www. ebi.ac.uk/Tools/msa/muscle/). Then, MEGA7 (Kumar et al. 2015) was first used to establish the best-fit model of evolution through the option "Find best DNA/ protein models" implemented in the program. Finally, a maximum likelihood tree with the appropriate options was constructed with 1000 bootstrap replicates. The gene structure of $S$. commersonii CBLs and CIPKs was analyzed using the gene structure display server (GSDS 2.0, http://gsds.cbi.pku.edu.cn/). Predictions of motifs were generated using Multiple Em for Motif Elicitation (MEME) program (http://meme-suite.org/tools/meme), with an optimum width of motifs ranging from 6 to 50, the maximum number of motifs set to 18 , and default values for other parameters. Palmitoylation sites were predicted by CSS-Palm 3.0 (http://csspalm.biocuckoo.org/) whereas myristoylation sites by Myristoylator (http://web.expasy. org/myristoylator/).

Gene duplications and evolutionary analysis: Tandem duplicated genes were defined as an array of two or more genes located on the same chromosome/scaffold and found within a $100 \mathrm{~kb}$ genomic window (Zhu et al. 2016). The locus search tool at the plant genome duplication database $P G G D$ (http://chibba.agtec.uga.edu/duplication/) was used to determine if $C I P K$ and $C B L$ genes existed within genomic duplication blocks whereas the CoGe platform (https://genomevolution.org/coge/) was used to confirm tandem duplications.

Public RNA-seq based expression analysis: We used fastq data related to four tissues (flower, leaf, tuber, and stolon) and deposited in the sequence read archive (SRA) under study $S R P 050412$ to analyze the expression profiles of $S c C B L s$ and $S c C I P K s$. Unmapped reads were considered 
for the next analysis and were mapped against the S. tuberosum genome (ITAG annotation v. 1). The Binary Alignment Map files were then analyzed using the CufflinksCuffquant software (v. 2.2.1) to assemble the aligned reads and to access transcriptome complexity. Expression values for each gene were estimated based on reads per kilobase of transcript per million mapped reads (RPKM) using the default options. Transcriptions of all ScCBL and ScCIPK were also characterized in potato leaves from plants that were subjected to various treatments including heat stress $\left(35^{\circ} \mathrm{C}\right.$ for $\left.24 \mathrm{~h}\right)$, salt stress $(50 \mathrm{mM} \mathrm{NaCl}$ for $24 \mathrm{~h})$, osmotic stress $(260 \mu \mathrm{M}$ mannitol for $24 \mathrm{~h}), 50 \mathrm{mM} \beta$-aminobutyric acid, $50 \mathrm{mMbenzothiadiazole,} 10 \mathrm{mM}$ indole-3-acetic acid, $50 \mathrm{mM}$ abscisic acid, $50 \mathrm{mM}$ gibberellic acid, and $50 \mathrm{mM}$ 6-benzylaminopurine. In particular, data were retrieved from the public $S$. tuberosum database available in literature (http://solanaceae.plantbiology.msu.edu). The RPKM values of $S$. commersonii orthologs in control and stress conditions were collected.

Network and protein interaction of $S$. commersonii CBLs and CIPKs: For the protein interaction network, high confidence experimental data of interacting CBL and CIPK proteins in Arabidopsis were constructed using STRING (http://string-db.org/) with a confidence value $>0.7$. The ortholog proteins of the determined interacting S. tuberosum proteins were identified in $S$. commersonii by reciprocal $B L A S T P$ analysis.

\section{Results}

Ten non-redundant $C B L$ and $26 C I P K$ genes were identified in $S$. commersonii, $S$. tuberosum, and $S$. chacoense (Table 1) suggesting that their loci were substantially conserved after their evolutionary divergence. The multiple aminoacids (aa) sequence alignment of CBL proteins along with AtCBLs indicate that the general structures of CBLs were highly conserved with all CBLs having EF hands similar to those of AtCBLs (data not shown). Similarly, the multiple sequence alignments of CIPK proteins show that all members contain an N-terminal catalytic kinase domain and a C-terminal regulatory domain, which are necessary and very important for protein-protein interactions (data not shown). Once identified, we annotated and named each CBL and CIPK according to their phylogeny and protein sequence similarities with those of Arabidopsis (Fig. 1). In this way, closely-related orthologous and paralogous pairs of CBLs and CIPKs were identified based also on a high bootstrap value. As far as $S$. commersonii $C B L s$ are concerned, two genes clustering on the same clade with $A t C B L 4$ were identified as $S c C B L 4 a$ (on scaffold27252) and $S c C B L 4 b$ (on scaffold3852). Two paralogs of $C B L 4$ were also identified in $S$. tuberosum (PGSC003DMP400016074 and PGSC0003DMP400034042) and $S$. chacoense (g9425.t1 and g21063.t1) (Fig. 1A) suggesting that $C B L 4$ might have been duplicated before the divergence of the potato species. Similarly to $S c C B L 4$, genemark C2860222_abinit_gene_0_8 and maker_scaffold12242_snap_gene_0_48 clustered with $A t C B \bar{L} 8$. Hence, we named them $S c C B L 8 a$ and $S c C B L 8 b$, respectively. Interestingly, two paralogs of $C B L 8$ were found only in $S$. commersonii whereas one member was found in $S$. tubersoum (PGSC0003DMP400035601) and in $S$. chacoense (g6456.t1). By contrast, two paralogs of CBL5 were identified only in the cultivated $S$. tubersoum but not in its wild relatives (Fig. $1 A$ ). The remaining $C B L s$ were named using the prefix-like based on clustering with Arabidopsis. As far as CIPKs are concerned, CIPK6, CIPK11, CIPK14, and CIPK20 had two paralogs in all the species analyzed (Fig. $1 B$ ). The CIPK3 also underwent duplication events. Indeed, two copies were found in $S$. commersonii and S. chacoense whereas S. tuberosum harbored only one copy. The remaining CIPKS were identified as single orthologs (Fig. 1B). Furthermore, four CIPKS from S.tuberosum, three from S. commersonii and two from $S$. chacoense were named using the prefixspecific because they did not cluster with AtCIPKs. Detailed information regarding domain composition and the number of orthologs and paralogs of all species are reported in Table 1 Suppl., Figs 1 Suppl. and 2 Suppl.

Once genes were identified, we surveyed duplication events (data not shown). Phylogenetic results were confirmed through this analysis. Indeed, two tandem duplications were detected in $S$. tuberosum and $S$. commersonii CBLs whereas one was identified in $S$. chacoense (data not shown). The gene pairs include $C B L 4 a / C B L 4 b$ in all the three genomes analyzed here, $C B L 5 a / C B L 5 b$ in $S$. tuberosum and $C B L 8 a / C B L 8 b$ in $S$. commersonii. In contrast, no segmental duplications of $C B L$ genes were detected. A higher number of tandem duplications events (four events in $S$. tuberosum and five in $S$. commersonii and $S$. chacoense) were detected among CIPKs. The gene pairs include CIPK6a/CIPK6b, $C I P K 11 a / C I P K 11 b$, and CIPK20a/CIPK20b in all the species analyzed whereas $C I P K 3 a / C I P K 3 b$ were found duplicated in $S$. commersonii and $S$. chacoense but not in S. tuberosum.

Intron/exon organization of all CBLs and CIPKs were analyzed to further investigate their structural features. In the case of $C B L s$, the number of exons among the three species was generally conserved. Indeed, $C B L 1$-like, $C B L 3$ like, CBL4a, CBL8a,CBL9-like, and CBL10 harbored the same number of exons in $S$. commersonii, S. tuberosum, and $S$. chacoense, respectively. By contrast, differences were identified in $C B L 4 b$ and $C B L 8 b$ (Fig. 2 Suppl.). For example, $C B L 4 b$ harbor 16 exons in $S$. commersonii and 8 in $S$. tuberosum and $S$. chacoense whereas $C B L 8 b$ had 12 exons in $S$. commersonii, 7 in $S$. tuberosum, and 8 in $S$. chacoense. The $C B L$ gene exon structure validated the protein length (Table 1). Indeed, $S c C B L 4 b$ (16 exons) encoded for a peptide of 426 aa whereas the orthologs in $S$. tuberosum and $S$. chacoense (8 exons) was 215 aa in length. Also, the product of $S c C B L 8 b$ was longer than StCBL8b and SchacoCBL8 (320 aa in S. commersonii, 196 aa in $S$. tuberosum, and 221 aa in $S$. chacoense) reflecting differences in their gene structure. By contrast, members belonging to the $S c C I P K$ family clustered into two different sub-groups (an intron-rich clade and an intron-poor clade) (data not shown). In particular, 18 CIPKs (CIPK4-like, 
CIPK5-like, CIPK6a, CIPK6b, CIPK7-like, CIPK11a, CIPK11b, CIPK14a, CIPK14b, CIPK16-like, CIPK20a, CIPK20b, CIPK22, CIPK24-like, CIPK2-like, CIPK specific1, ScCIPK_specific2, ScCIPK_specific3) belong to the former group whereas eight ScCIPKS (CIPK17-like, CIPK1-like, CIPK23, CIPK24, CIPK3a, CIPK3b, CIPK8, and $C I P K 9$ ) belong to the latter. All motifs found were highly conserved in all species (data not shown). Similar to
AtCBLs, CBLs possessed a conserved serine residue in the PFPF motif, which is phosphorylated by CIPKs (Du et al. 2011) whereas all CIPKs contained conserved N-terminal catalytic kinase and C-terminal regulatory domain. Furthermore, searching for other possible motifs that could be functionally important in fulfilling CBL roles, we found that CBL1-like, CBL4a, CBL4b and CBL9 harbored a conserved $\mathrm{N}$-myristoylation motif (MGXXXS/T) that

Table 1. Features of calcineurin B-likes (CBLs) and CBL-interacting kinases (CIPKs) identified in Solanum commersonii, S. tuberosum, and $S$. chacoense. Strand and protein lengths (the number of amino acids) are reported. N/A - means not assigned.

\begin{tabular}{|c|c|c|c|c|c|c|}
\hline Gene/protein & $\begin{array}{l}\text { S. commersonii } \\
\text { strand }\end{array}$ & length & $\begin{array}{l}\text { S. tuberosum } \\
\text { strand }\end{array}$ & length & $\begin{array}{l}\text { S. chacoense } \\
\text { strand }\end{array}$ & length \\
\hline CBL1-like & - & 214 & - & 213 & - & 223 \\
\hline CBL2-like & + & 223 & - & 247 & + & 220 \\
\hline CBL3-like & + & 225 & + & 262 & - & 225 \\
\hline CBL4a & - & 215 & + & 200 & + & 215 \\
\hline$C B L 4 b$ & - & 215 & - & 426 & + & 215 \\
\hline CBL5a & - & 120 & + & 109 & + & 120 \\
\hline CBL5b & & 99 & N/A & N/A & $\mathrm{N} / \mathrm{A}$ & N/A \\
\hline CBL8a & $\mathrm{N} / \mathrm{A}$ & N/A & - & 159 & - & 221 \\
\hline CBL8b & + & 196 & - & 320 & + & 196 \\
\hline CBL9-like & + & 262 & - & 215 & - & 214 \\
\hline CBL10 & + & 291 & + & 294 & - & 258 \\
\hline \multicolumn{7}{|l|}{ CIPK } \\
\hline CIPK1-like & + & 457 & + & 279 & + & 457 \\
\hline CIPK2-like & - & 447 & - & 474 & - & 479 \\
\hline CIPKЗа & + & 439 & + & 546 & + & 438 \\
\hline CIPK3b & N/A & $\mathrm{N} / \mathrm{A}$ & + & 439 & - & 475 \\
\hline CIPK4-like & + & 438 & + & 437 & + & 438 \\
\hline CIPK5-like & - & 451 & + & 450 & + & 451 \\
\hline CIPK5b & N/A & $\mathrm{N} / \mathrm{A}$ & N/A & N/A & + & 451 \\
\hline CIPK6a & - & 433 & + & 305 & - & 433 \\
\hline CIPK6b & - & 425 & + & 424 & + & 402 \\
\hline CIPK7-like & - & 434 & - & 433 & - & 434 \\
\hline CIPK8 & - & 448 & + & 468 & + & 448 \\
\hline CIPK9 & - & 409 & - & 406 & - & 452 \\
\hline CIPK11a & - & 420 & - & 419 & - & 420 \\
\hline CIPK11b & + & 447 & - & 1006 & + & 446 \\
\hline CIPK14-like & - & 451 & + & 450 & - & 451 \\
\hline CIPK16-like & + & 488 & + & 487 & - & 488 \\
\hline CIPK17-like & - & 532 & + & 425 & + & 471 \\
\hline CIPK20a & - & 459 & - & 461 & - & 463 \\
\hline CIPK20b & - & 371 & - & 286 & - & 221 \\
\hline CIPK22-like & - & 437 & - & 436 & + & 437 \\
\hline CIPK23 & - & 456 & - & 444 & - & 456 \\
\hline CIPK24 & + & 447 & + & 564 & + & 447 \\
\hline CIPK25 & - & 457 & - & 451 & - & 457 \\
\hline CIPK_specific1 & - & 471 & + & 447 & - & 471 \\
\hline CIPK_specific2 & + & 444 & + & 443 & + & 444 \\
\hline CIPK_specific3 & + & 442 & + & 153 & N/A & N/A \\
\hline CIPK_specific4 & - & 446 & $\mathrm{~N} / \mathrm{A}$ & $\mathrm{N} / \mathrm{A}$ & $\mathrm{N} / \mathrm{A}$ & $\mathrm{N} / \mathrm{A}$ \\
\hline
\end{tabular}




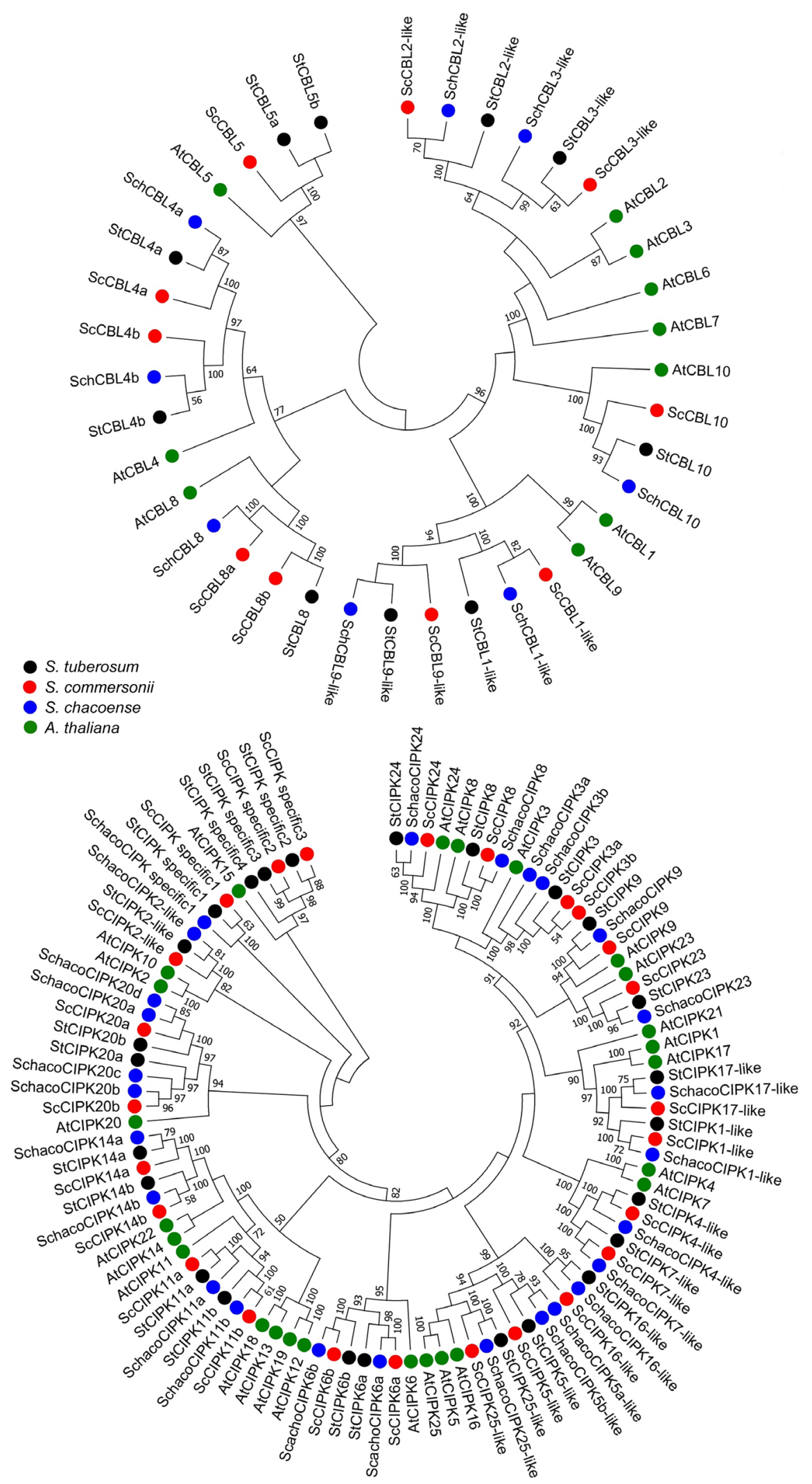

Fig. 1. Phylogenetic analysis of calcineurin B-like (CBL, upper) and CBL-interacting kinase (CIPK, bottom) gene families in Solanum commersonii (red), S. chacoense (blue), S. tuberosum (black), and Arabidopsis thaliana (green). Full length protein sequences of CBLs and CIPKs were used to construct maximum likewood trees by using the MEGA7.0 software with a bootstrap value of 100 (only values $>50$ are shown). 


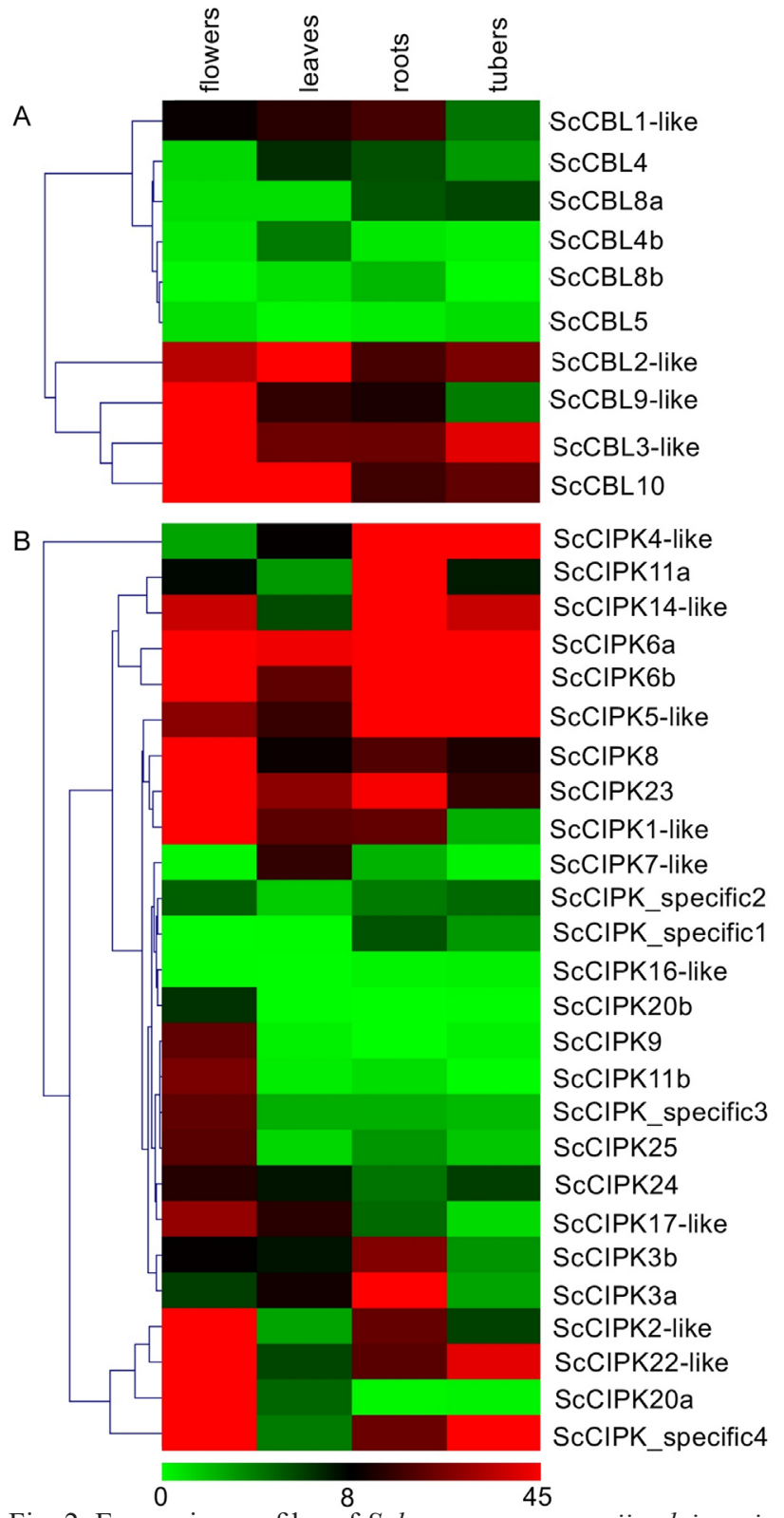

Fig. 2. Expression profiles of Solanum commersonii calcineurin $B$-like $(S c C B L, A)$ and $S$. commersonii $C B L$-interacting kinase $(S c C I P K, B)$ genes in four tissues (flowers, leaves, roots, and tubers). Data are reported as reads per kilobase of transcript per million mapped reads.

might function in membrane targeting of the CBLs.

Expression patterns of $S c C B L s$ and $S c C I P K S$ based on RNAseq data from four $S$. commersonii tissues (flowers, leaves, roots, and tubers) are reported as a heatmap in Fig. 2. The ScCBL1-like, ScCBL2-like, ScCBL3-like, ScCBL9like, and $S c C B L 10$ were highly expressed throughout the various tissues in contrast to the others, which exhibited low expressions. In particular, ScCBL3-like, ScCBL9-like, and $S c C B L 10$ showed the highest expression in flowers whereas $S c C B L 2$-like was highly expressed in leaves and ScCBL4-like in roots.

As far as $S c C I P K s$ is concerned, four different groups were identified based on their expression patterns (Fig. $4 B$ ). The first group comprised seven ScCIPKs (ScCIPK1like, ScCIPK5-like, ScCIPK6a, ScCIPK6b, ScCIPK8, ScCIPK14-like, and ScCIPK23), which were highly expressed in all tissues analyzed, whereas the second group included ScCIPKs with a low abundancy (ScCIPK7like, ScCIPK specific1, ScCIPK_specific2, ScCIPK16like, and $S c \bar{C} I P K 20 b)$. The third group included five ScCIPKs (ScCIPK9, ScCIPK11b, ScCIPK20b, ScCIPK25, and ScCIPK_specific3), which showed expressions in flowers but not in the other tissues. The fourth group of $S c C I P K s$ contained those highly expressed in all tissues excepted in leaves (ScCIPK2-like, ScCIPK20a, ScCIPK22-like, and ScCIPK_specific4). Only ScCIPK4like and ScCIPK11a showed a high expression in roots but not in the other tissues. Collectively, these data indicate that $S$. commersonii CBLs and CIPKs might participate in specific functions in different organs.

Subsequently, we wondered whether CBLs and CIPKs play roles under different stresses. To date expression data on $S$. commersonii tissues exposed to any stress are not available. Since orthologous genes are known to retain equivalent functions in different organisms, we retrieved CBLs and CIPKs RPKM values from $S$. tuberosum experiments involving several stresses. As shown in Fig. 3, transcriptions of many $C B L$ and $C I P K$ genes altered in response to the various treatments, by contrast, only CBL5, CIPK9, and CIPK16 did not change their transcriptional activity following stress conditions. $C B L 1-$ like, CBL3-like, CBL9-like, CBL10, and CIPK17 increased their expression following heat treatment whereas the expressions of CBL10, CIPK2, CIPK5, CIPK20a, $C I P K 11 a$ were altered in both salt conditions and mannitol conditions. The CBL2-like, CBL10, CIPK1-like, CIPK3, CIPK4-like, CIPK6a, CIPK6b, CIPK8, CIPK14-like, and CIPK24 responded to 6-benzylaminopurine treatment whereas the other hormone treatments did not affect the expression of either CBLs or CIPKs (Fig. 3).

Further analysis will be carried out to assess the role of $C B L$ and $C I P K$ genes in $S$. commersonii challenged to abiotic and biotic stress conditions. The CIPKs have been demonstrated to interact with CBLs and several transporters to mediate multiple biological processes (Yang et al. 2008, Held et al. 2011, Huertas et al. 2012). Therefore, to further understand the interactions between ScCBL and ScCIPK proteins, an interaction network was built using the on-line tool STRING (Fig. 4). The homologous proteins with the highest bit score were considered as STRING queries. Using this approach, a total of 184 different interactions were found with an average of 13.6 for each protein pairs. The putative CIPK partners include CBLs involved in calcium ion binding, Arabidopsis $\mathrm{K}^{+}$transporter (AKT), salt overly sensitive 1 (SOS1) related to sodium-hydrogen transport, abscisic acid-insensitive 1-2 (ABI1 and ABI2) involved in protein dephosphorylation, calcium exchanger 1 (CAX1) related to calcium ion transport, and other proteins with binding activity. Five different CIPKs (CIPK1-like, CIPK3, CIPK7-like, CIPK9, and CIPK23) were found to putatively interact widespread with CBL1-like, CBL2like, CBL3-like, CBL9, and CBL10 whereas CIPK5-like 


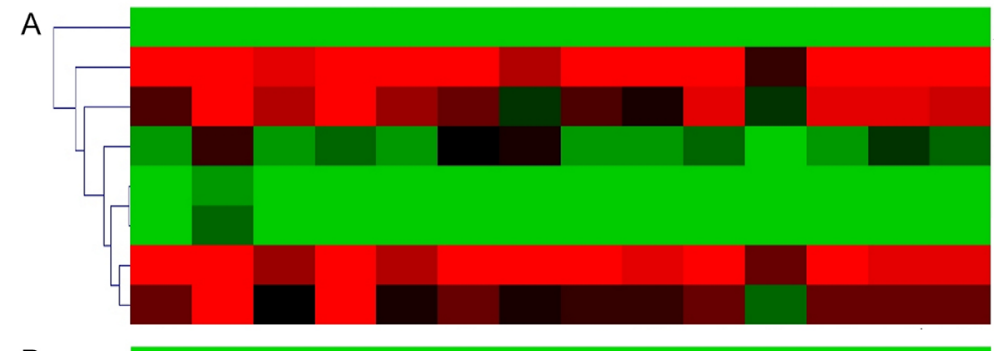

StCBL5

StCBL2

StCBL1

StCBL9

$\mathrm{StCBL4}$

$\mathrm{StCBL8}$

StCBL3

B

StCBL10

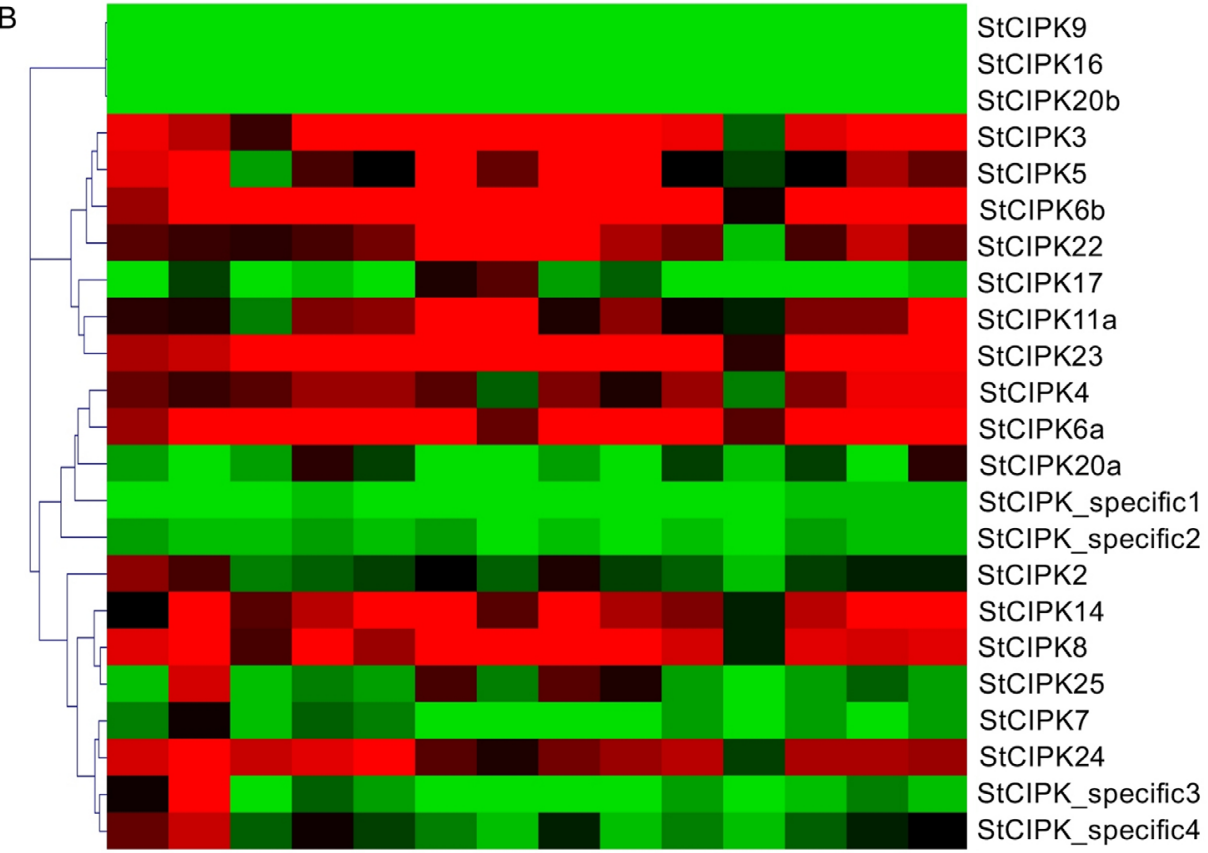

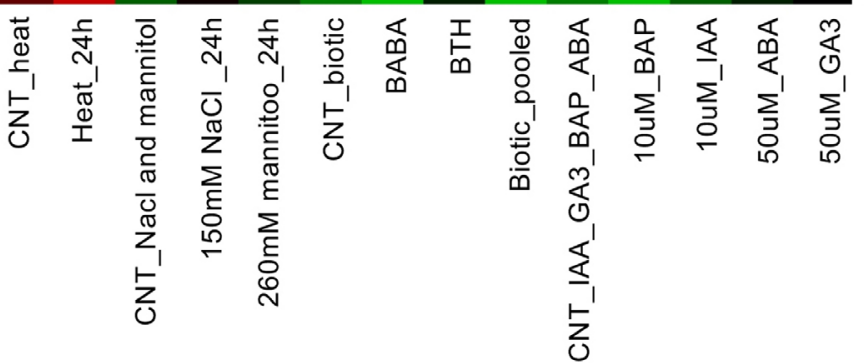

Fig. 3. Expression profiles of Solanum commersonii calcineurin B-like (StCBL) $(A)$ and $S$. commersonii CBL-interacting kinase $(\operatorname{StCIPK})(B)$ genes in response to heat, salt, mannitol, $\beta$-aminobutyric acid (BABA), benzothiadiazole (BTH), 6-benzylaminopurine (BAP), control (CNT), indole-3-acetic acid (IAA), abscisic acid (ABA), and gibberellic acid $\left(\mathrm{GA}_{3}\right)$ tretments.

and CIPK14-like are predicted to interact specifically with CBL2-like and CBL9and CIPK20 interact only with CBL9. Previous functional analyses have clarified that these interactions may participate in the regulation of plant ions transport, developmental processes, hormone signaling, and abiotic stress responses (Zhang et al. 2014). However, further molecular analyses are needed to clarify these interactions in S. commersonii.

\section{Discussion}

Calcium ions play a crucial role as second messengers in mediating various adaptive responses in plants under environmental stresses (Tuteja et al. 2007, Edel et al.
2017). Transient $\mathrm{Ca}^{2+}$ changes are sensed by several $\mathrm{Ca}^{2+}$ binding proteins. Among them, the complex CBL-CIPK is the most interesting in transmitting stress signals from outside of the cell cytosol and to the nucleus. Despite extensive studies are available on the CBL-CIPK network in plant models, such as Arabidopsis, rice, and maize (Kolukisaoglu et al. 2004, Zhang et al. 2016), there are no reports regarding a comprehensive analysis of these two gene families in potato.

In the present study, $10 C B L$ genes were identified in the genome of the cultivated potato $S$. tuberosum and in its wild relatives $S$. commersonii and $S$. chacoense. A previous study by Mohanta et al. (2015) already reported the presence of many $C B L s$ in $S$. tuberosum. However, details and clues regarding their structure and evolution 


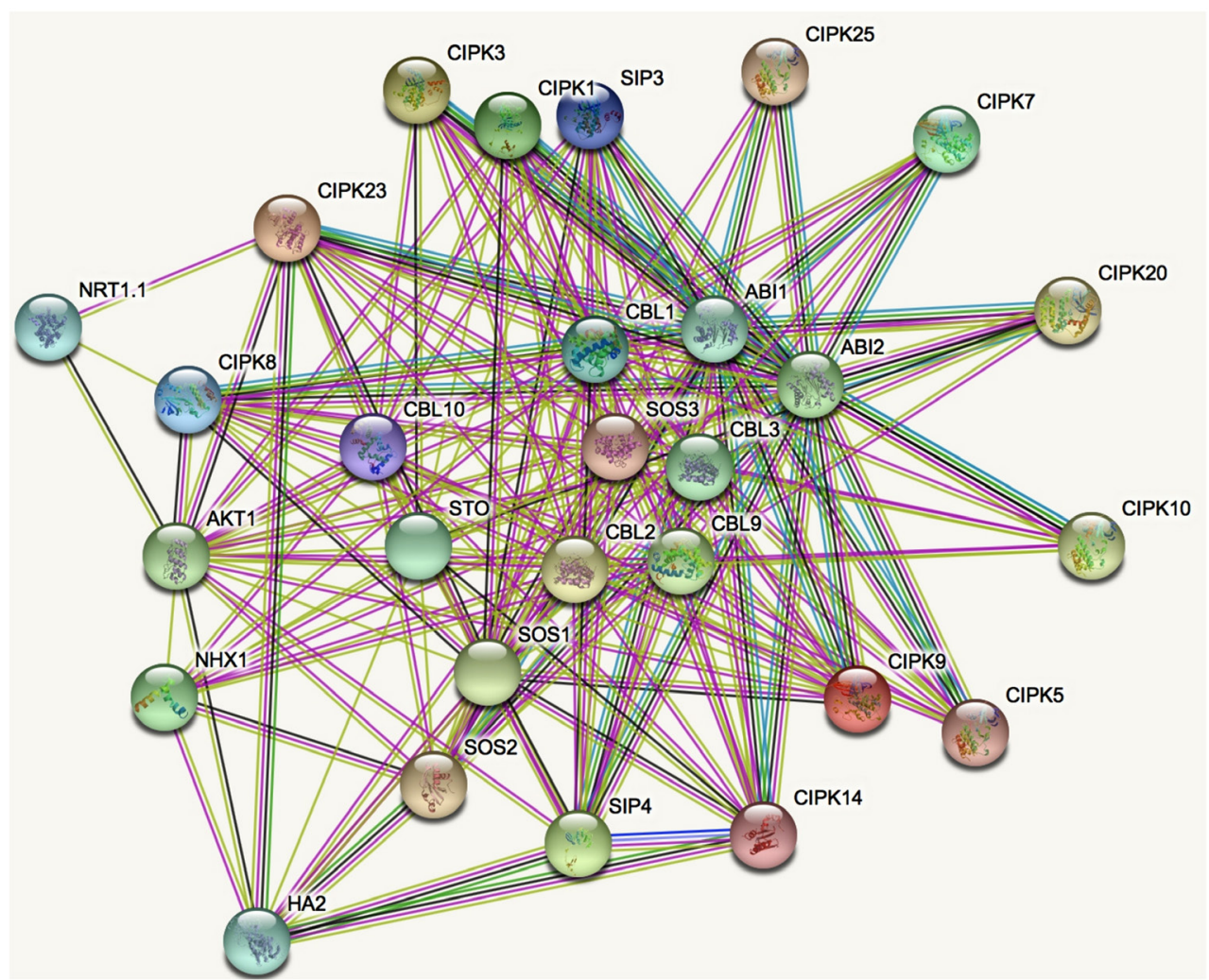

Fig. 4. A predicted interaction network of calcineurin B-like (CBLs) and CBL-interacting kinases (CIPKs) in Solanum commersonii. The CBL4 and CIPK24 are reported as salt overly sensitive (SOS) 3 and SOS2, respectively.

are lacking. Ten CBLs were also identified in Arabidopsis, rice, and poplar (Kolukisaoglu et al. 2004, Yu et al. 2007). To date, the highest number of CBLs (13) has been identified in Gossypium raimondii (Mohanta et al. 2015) whereas the smallest (6) in Sorghum bicolor (Weinl et al. 2009). Our results on CIPKs confirm that this gene family in plants is larger compared to $C B L$. Indeed, we identified $26 C I P K s$ in the three potato genomes analyzed, 20, 26, and 30 CIPKs were found in Vitis, Arabidopsis, and rice, respectively (Kolukisaoglu et al. 2004, Kanvar et al. 2014, Xi et al. 2017). These findings indicate that gene duplication events, such as segmental and/or tandem duplications might play a significant role in their expansion. For such reasons, once genes were identified, we analyzed the relative contributions of both tandem duplications and segmental duplications. These mechanisms represent the two major forces driving gene evolution (Kong et al. 2007). Our results indicate that both $C B L$ and $C I P K$ genes originate from tandem duplication events. In contrast, $\mathrm{Xi}$ et al. (2017) reported that $50 \%$ of $V v C B L s$ and $40 \%$ of $V v C I P K s$ arise by tandem duplication, and $25 \%$ of $V v C I P K s$ are derived from segmental duplications in $V$. vinifera. Our phylogenetic analysis provided evidence that variability in the number of paralogs within different potato species were present. For example, CIPK3 had two paralogs in $S$. commersonii and $S$. chacoense but only one in $S$. tuberosum hinting that the cultivated potato might have lost a copy due to domestication events, as already reported Lam et al. (2010) in G. max. By contrast, two paralogs of CIPK5 were identified in S. chacoense but not in the other species, underlining the idea that, in different plant species, the number of copies might expand through different mechanisms. As far as structural features are concerned, such as conserved motifs and binding domains in S. commersonii, our results are in line with those reported in Arabidopsis and other species (Kolukisaoglu et al. 2004, Lyzenga et al. 2013, Yu et al. 2014, Mohanta et al. 2015). For example, the recently characterized motif PFPF, which is important for the phosphorylation cascades of CBLs (Ames et al. 1997, Kanwar et al. 2014), was identified in all ScCBL protein sequences. In addition, searching other possible motifs that could be functionally important in fulfilling CBL roles, we found that 4 out of $10 \mathrm{ScCBLs}$ harbor a conserved N-myristoylation motif (MGXXXS/T), whereas 8 out of 10 bear a typical palmitoylation site at the $\mathrm{N}$-terminal. A recent paper by Saito et al. (2018) revealed that these modifications promote a stable membrane association of soluble proteins including CBLs. For example, plasma membrane targeting CBL1 and CBL9 in Arabidopsis requires a dual lipid modification by N-myristoylation and S-acylation (Batistic et al. 2008). N-myristoylation and S-acylation of CBL4 are also essential for regulation of the $\mathrm{Na}^{+} / \mathrm{H}^{+}$antiporter salt overly sensitive 1 (SOS1) and the potassium channel Arabidopsis $\mathrm{K}^{+}$transporter 2 (AKT2) (Ishitani et al. 2000, Qiu et al. 2002, Held et al. 2011). These findings confirm an important role of dual lipid modifications of regulatory soluble proteins for the modulation of intrinsic 
membrane protein activity. However, the requirement of dual lipid modifications for $\mathrm{Ca}^{2+}$-regulated processes in general remains to be elucidated, and how widespread this targeting mechanism is in plant signaling processes remains to be determined.

The expression profile of a gene in a specific plant tissue provides clues related to its function under a particular conditions or a stage of growth and development. Using tissue-specific transcriptome analysis, Lu et al. (2018) identified key genes with different expressions in individual organs involved in de novo fatty acid biosynthesis that affect lipid metabolism and oil content in Brassica napa seeds. In the case of $C B L s$, it has been shown through promoter- $\beta$-glucuronidase activity that $C B L 1$ and $C B L 9$ are expressed in leaves, roots, flowers, and vascular tissues in Arabidopsis (Pandey et al. 2004, Cheong et al. 2007) whereas $C B L 4$ is mainly expressed in root tissue (Quan et al. 2007) and CBL10 in the shoot tissue (Cheong et al. 2010). In our study, ScCBL1-like and ScCBL9-like confirmed high expressions throughout various tissues suggesting that these genes might have a similar function in different plant tissues. By contrast, $S c C B L 10$ was mainly expressed in flowers and ScCBL4 in roots, indicating diverse functions for their products in plant growth and development. Our data are also in line with those showed by Shi et al. (2000) in Arabidopsis. In the case of CIPKs, we found that many ScCIPKs (i.e., ScCIPK1-like, ScCIPK5like, ScCIPK6a, ScCIPK6b, ScCIPK8, ScCIPK14-like, and $S c C I P K 23$ ) were constitutively expressed in all tissues analyzed indicating that they may play widespread roles in these organs as already shown for other species such as Triticum aestivum and Manihot esculenta (Sun et al. 2015, Mo et al. 2018). By contrast, other CIPK genes were abundantly expressed in certain tissues. For example, ScCIPK4 was mainly expressed in roots, as also reported Linn et al. (2017) in Arabidopsis whereas the expression of ScCIPK25 was detected in flowers and roots, consistent with data by Meena et al. (2018).

Previous studies demonstrated that the CBLCIPK complex is involved in regulating response to environmental stresses (Kanwar et al. 2014, Yu et al. 2014). For example, AtCBL10 was reported to interact with AtCIPK24 in response to salt stress (Kim et al. 2007) and was shown to negatively compete with AtCIPK23 for binding to Arabidopsis $\mathrm{K}^{+}$transporter (AKT1), modulating its activity (Huertas et al. 2012, Ren et al. 2013). Our dataset analysis revealed that $S c C B L 10$ (orthologous of $A t C B L 10)$ together with $S c C B L 3$ and $S c C B L 9$ increased its expression following heat stress. The $C B L 3$ has been reported to be induced also in rice upon heat stress (Mohanta et al. 2015) whereas CBL9 was activated in Brassica napus (Zhang et al. 2014) following the same stress conditions. These results reinforce the hypothesis that more than one $C B L$ might participate in regulating the same stress conditions. Other stress responsive $C B L s$ are CBL5 and CBL4. Indeed, in 2010, Cheong et al. (2010) provided evidence that $A t C B L 5$ confers drought tolerance whereas Qiu et al. (2002) showed that AtCBL4 is involved in salt tolerance. In contrast to these results, ortholog genes ScCBL4 and ScCBL5 did not change their expressions under all the stress conditions studied here. These data support that CBL/CIPK signaling components may have different functions in responding stress conditions in different plant species.

\section{Conclusions}

In the present study, we reported for the first time a genome wide analysis of CBLs and CIPKs in S. tuberosum, $S$. commersonii, and $S$. chacoense. The identification of $10 C B L s$ and $26 C I P K s$ suggests that the complexity of the CBL-CIPK signaling network in potato is similar to that reported in other species. The analysis of evolutionary features of these genes revealed that tandem duplication events were the main evolutionary forces underlying the expansion of both gene families. Finally, the investigation of the role of these genes upon stress conditions pointed out that $S$. commersonii $C B L s$ and $C I P K s$ exhibited differential responses to multiple stimuli. All these findings increase our understanding on the calcium signal pathways in potatoes, paving the way for future breeding approaches.

\section{References}

Ames, J.B., Ishima, R., Tanaka, T., Gordon, J.I., Stryer, L., Ikura, M.: Molecular mechanics of calcium-myristoyl switches. Nature 389: 198-202, 1997.

Aversano, R., Contaldi, F., Ercolano, M. R., Grosso, V., Iorizzo, M., Tatino, F., Xumerle, L., Dal Molin, A., Avanzato, C., Ferrarini, M., Delledonne, M., Sanseverino, W., Aiese Cigliano, R., Capella-Gutierrez, S., Gabaldón, T., Frusciante, L., Bradeen, J.M., Carputo, D.: The Solanum commersonii genome sequence provides insights into adaptation to stress conditions and genome evolution of wild potato relatives. Plant Cell 27: 954-968, 2015.

Aversano, R., Contaldi, F., Adelfi, M.G., D'Amelia, V., Diretto, G., De Tommasi, N., Vaccaro, C., Vassallo, A., Carputo, D.: Comparative metabolite and genome analysis of tuber-bearing potato species. - Phytochemistry 137: 42-51, 2017.

Batistic, O., Sorek, N., Schultke, S., Yalovsky, S., Kudla, J.: Dual fatty acyl modification determines the localization and plasma membrane targeting of $\mathrm{CBL} / \mathrm{CIPK} \mathrm{Ca}^{2+}$ signaling complexes in Arabidopsis. - Plant Cell 20: 1346-1362, 2008.

Carputo, D., Aversano, R., Barone, A., Di Matteo, A., Iorizzo, M., Sigillo, L., Zoina, A., Frusciante, L.: Resistance to Ralstonia solanacearum of sexual hybrids between Solanum commersonii and S. tuberosum. - Amer. J. Potato Res. 86: 196-202, 2009.

Carputo, D., Alioto, D., Aversano, R., Garramone, R., Miraglia, V., Villano, C., Frusciante, L.: Genetic diversity among potato species as revealed by phenotypic resistances and SSR markers. - Plant Genet. Resour. 11: 131-139, 2013.

Chen, X., Gu, Z., Xin, D., Hao, L., Liu, C., Huang, J., Ma, B., Zhang, H.: Identification and characterization of putative CIPK genes in maize. - J. Genet. Genomics 38: 77-87, 2011.

Cheng, S.H., Willmann, M.R., Chen, H., Sheen, J.: Calcium signaling through protein kinases: the Arabidopsis calciumdependent protein kinase gene family. - Plant Physiol. 129: 469-485, 2002.

Cheong, Y.H., Pandey, G.K., Grant. J.J., Batistic, O., Li, L., Kim, B.G., Lee, S.C., Kudla, J., Luan, S.: Two calcineurin 
B-like calcium sensors, interacting with protein kinase CIPK23, regulate leaf transpiration and root potassium uptake in Arabidopsis. - Plant. J. 52: 223-239, 2007.Cheong, Y.H., Sung, S.J., Kim, B.G., Pandey, G.K., Cho, J.S., Kim, K.N., Luan, S.: Constitutive overexpression of the calcium sensor CBL5 confers osmotic or drought stress tolerance in Arabidopsis. - Mol. Cells 29: 159-165, 2010.

D'Amelia, V., Aversano, R., Batelli, G., Caruso, I., Castellano Moreno, M., Castro-Sanz, A.B., Chiaiese, P., Fasano, C., Palomba, F., Carputo, D.: High AN1 variability and interaction with basic helix-loop-helix co-factors related to anthocyanin biosynthesis in potato leaves. - Plant J. 80: 527-540, 2014.

D'Amelia, V., Aversano, R., Ruggiero, A., Batelli, G., Appelhagen, I., Dinacci, C., Hill, L., Martin, C., Carputo, D.: Subfunctionalization of duplicate $M Y B$ genes in Solanum commersonii generated the cold-induced ScAN2 and the anthocyanin regulator ScAN1. - Plant Cell Environ. 41:1038$1051,2018$.

Du, W., Lin, H., Chen, S., Wu, Y., Zhang, J., Fuglsang, A.T., Palmgren, M.G., Wu, W., Guo, Y.: Phosphorylation of SOS3like calcium-binding proteins by theirinteracting SOS2like protein kinases is a common regulatory mechanism in Arabidopsis. - Plant Physiol. 156: 2235-2243, 2011.

Edel, K.H., Marchadier, E., Brownlee, C., Kudla, J., Hetherington, A.M.: The evolution of calcium-based signaling in plants. Curr. Biol. 27: R667-R679, 2017.

Esposito, S., Aversano, R., D’Amelia, V., Villano, C., Alioto, D., Mirouze, M., Carputo, D.: Dicer-like and RNA-dependent $R N A$ polymerase gene family identification and annotation in the cultivated Solanum tuberosum and its wild relative $S$. commersonii. - Planta 248: 729-743, 2018 a.

Esposito, S., Aversano, R., Bradeen, J.M., Di Matteo, A., Villano, C., Carputo, D.: Deep-sequencing of Solanum commersonii small RNA libraries reveals riboregulators involved in cold stress response. - Plant Biol. doi: 10.1111/plb.12955, $2018 \mathrm{~b}$.

Hanneman, R.E., Bamberg, J.B.: Inventory of Tuber-Bearing Solanum Species. - Research Division of the College of Agriculture and Life Sciences, University of Wisconsin, Madison 1986

Hawkes, J.G.: The potato: evolution, biodiversity and genetic resources. - Amer. Potato J. 67: 733-735, 1990.

Held, K., Pascaud, F., Eckert, C., Gajdanowicz, P., Hashimoto, K., Corratge-Faillie, C., Offenborn J.N., Lacombe, B., Dreyer, I., Thibaud, J.B., Kudla, J.: Calcium-dependent modulation and plasma membrane targeting of the AKT2 potassium channel by the CBL4/CIPK6 calcium sensor/protein kinase complex. - Cell Res. 21: 1116-1130, 2011.

Huertas, R., Olias, R., Eljakaoui, Z., Galvez, F. J., Li, J., De Morales, P.A., Belver, A., Rodriguez-Rosales, M.P.: Overexpression of SISOS2 (SlCIPK24) confers salt tolerance to transgenic tomato. - Plant Cell Environ. 35: 1467-1482, 2012.

Ishitani, M., Liu, J., Halfter, U., Kim, C.S., Shi, W., Zhu, J.K.: SOS3 function in plant salt tolerance requires $\mathrm{N}$-myristoylation and calcium binding. - Plant Cell 12: 1667-1678, 2000.

Kanwar, P., Sanyal, S.K., Tokas, I., Yadav, A.K., Pandey A., Kapoor S., Pandey, G.K.: Comprehensive structural, interaction and expression analysis of CBL and CIPK complement during abiotic stresses and development in rice. - Cell Calcium 56: 81-95, 2014.

Kim, S.Y., Kim, S.G., Kim, Y.S., Seo, P.J., Bae, M., Yoon, H.K., Park, C.M.: Exploring membrane-associated NAC transcription factors in Arabidopsis: implications for membrane biology in genome regulation. - Nucl. Acids Res. 35: 203-213, 2007.

Kolukisaoglu, U., Weinl, S., Blazevic, D., Batistic, O., Kudla,
J.: Calcium sensors and their interacting protein kinases: genomics of the Arabidopsis and rice CBL-CIPK signaling networks. - Plant Physiol. 134: 43-58, 2004.

Kong, H., Landherr, L.L., Frohlich, M.W., Leebens-Mack, J., Ma, H., De Pamphilis, C.W.: Patterns of gene duplication in the plant $S K P 1$ gene family in angiosperms: evidence for multiple mechanisms of rapid gene birth. - Plant J. 50: 873885, 2007.

Kudla, J., Xu, Q., Harter, K., Gruissem, W., Luan, S.: Genes for calcineurin B-like proteins in Arabidopsis are differentially regulated by stress signals. - Proc. nat. Acad. Sci. USA 96: 4718-4723, 1999.

Kumar, S., Stecher, G., Tamura, K.: MEGA7: molecular evolutionary genetics analysis version 7.0. - Mol. Biol. Evol. 33: 1870-1874, 2015.

Li, J., Jiang, M., Ren, L., Liu, Y., Chen, H.: Identification and characterization of $C B L$ and $C I P K$ gene families in eggplant (Solanum melongena L.). - Mol. Genet. Genom. 291: 17691781,2016

Lam, H.M., Xu, X., Liu, X., Chen, W., Yang, G., Wong, F.L., Li, M.W., He, W., Qin, N., Wang, B., Li, J., Jian, M., Wang, J., Shao, G., Wang, J., Sun, S.S., Zhang, G.: Resequencing of 31 wild and cultivated soybean genomes identifies patterns of genetic diversity and selection. - Nat. Genet 42: 1053-1059, 2010.

Linn, J., Ren, M., Berkowitz, O., Ding, W., Van der Merwe, M.J.,Whelan, J., Jost, R.: Root cell-specific regulators ofphosphate-dependent growth. - Plant Physiol. 174: 19691989, 2017.

Larkin, M.A., Blackshields, G., Brown, N.P., Chenna, R., McGettigan, P.A., McWilliam, H., Valentin, F., Wallace, I.M., Wilm, A., Lopez, R., Thompson, J.D., Gibson, T.J., Higgins, D.G.: Clustal W and Clustal X version 2.0. - Bioinformatics 23: 2947-2948, 2007.

Lu, S., Sturtevant, D., Aziz, M., Jin, C., Li, Q., Chapman, K. and Guo, L.: Spatial analysis of lipid metabolites and expressed genes reveals tissue-specific heterogeneity of lipid metabolism in high- and low-oil Brassica napus L. seeds. Plant J. 94: 915-932, 2018.

Lyzenga, W.J., Liu, H.X., Schofield, A., Muise-Hennessey, A., Stone, S.L.: Arabidopsis CIPK26 interacts with KEG, components of the ABA signalling network and is degraded by the ubiquitin-proteasome system. - J. exp. Bot. 64: 27792791, 2013.

Machida-Hirano, R.: Diversity of potato genetic resources. Breed. Sci. 65: 26-40, 2015.

Mao, J., Manik, S.M.N., Shi, S., Chao, J., Jin, Y., Wang, Q., Liu, H.: Mechanisms and physiological roles of the CBL-CIPK networking system in Arabidopsis thaliana. - Genes 7: E62, 2016.

Marchler-Bauer, A., Lu, S., Anderson, J.B., Chitsaz, F., Derbyshire, M.K., De Weese-Scott, C., Fong, J.H., Geer, L.Y., Geer, R.C., Gonzales, N.R., Gwadz, M., Hurwitz, D.I., Jackson, J.D., Ke Z., Lanczycki, C.J., Lu, F., Marchler, G.H., Mullokandov, M., Omelchenko, M.V., Robertson, C.L., Song, J.S., Thanki, N., Yamashita, R.A., Zhang, D., Zhang N., Zheng, C., Bryant, S.H: CDD: a conserved domain database for the functional annotation of proteins. - Nucl. Acids Res. 39: D225-D229, 2011.

Meena, M.K., Vishwakarma, N.K., Tripathi, V., Chattopadhyay, D.: CBL-interacting protein kinase 25 contributes to root meristem development. - J. exp. Bot. 70: 133-147, 2018.

Micheletto, S., Boland, R., Huarte, M.: Argentinian wild diploid Solanum species as sources of quantitative late blight resistance. - Theor. sppl. Genet. 101: 902-906, 2000.

Mo, C., Wan, S., Xia, Y., Ren, N., Zhou, Y., Jiang, X.: Expression 
patterns and identified protein-protein interactions suggest that cassava CBL-CIPK signal networks function in responses to abiotic stresses. - Front. Plant Sci. 9: 269, 2018.

Mohanta, T.K., Mohanta, N., Mohanta, Y.P., Parida, H.B. Genome-wide identification of Calcineurin B-Like $(C B L)$ gene family of plants reveals novel conserved motifs and evolutionary aspects in calcium signaling events. - BMC Plant Biol. 15: 189, 2015.

Mori, K., Asano, K., Tamiya, S., Nakao, T., Mori, M.: Challenges of breeding potato cultivars to grow in various environments and to meet different demands. - Breed. Sci. 65: 3-16, 2015.

Palta, J.P., Simon, G.: Breeding potential for improvement of freezing stress resistance: genetic separation of freezing tolerance, freezing avoidance, and capacity to cold acclimate. - In: Li, P.H. (ed.): Advances in Plant Cold Hardiness. Pp. 299-310. CRC Press, Boca Raton 1993.

Pandey, G.K., Cheong, Y.H., Kim, K.N., Grant, J.J., Li, L., Hung, W., D’Angelo, C., Weinl, S., Kudla, J., Luan, S.: The calcium sensor calcineurin B-like 9 modulates abscisic acid sensitivity and biosynthesis in Arabidopsis. - Plant Cell 16: 1912-1924, 2004.

Pandey, G.K., Kanwar, P., Singh, A., Steinhorst, L., Pandey, A., Yadav, A.K., Tokas, I., Sanyal, S.K., Kim, B.G., Lee, S.C.: Calcineurin B-like protein-interacting protein kinase CIPK21 regulates osmotic and salt stress responses in Arabidopsis. Plant Physiol. 169: 780-792, 2015.

Qiu, Q.S., Guo, Y., Dietrich, M.A., Schumaker, K.S., Zhu, J.K.: Regulation of SOS1, a plasma membrane $\mathrm{Na}^{+} / \mathrm{H}^{+}$exchanger in Arabidopsis thaliana, by SOS2 and SOS3. - Proc. nat. Acad. Sci. USA 99: 8436-8441, 2002

Quan, R., Lin, H., Mendoza, I., Zhang, Y., Cao, W., Yang, Y., Shang, M., Chen, S., Pardo, J.M., Guo, Y.: SCABP8/CBL10, a putative calcium sensor, interacts with the protein kinase SOS2 to protect Arabidopsis shoots from salt stress. - Plant Cell 19: 1415-1431, 2007.

Ren, X.L., Qi, G.N., Feng, H.Q., Zhao, S., Zhao, S.S., Wang, Y., Wu, W.H.: Calcineurin B-like protein CBL10 directly interacts with AKT1 and modulates $\mathrm{K}^{+}$homeostasis in Arabidopsis. - Plant J. 74: 258-266, 2013.

Saito, S., Hamamoto, S., Moriya, K., Matsuura, A., Sato, Y., Muto, J., Noguchi, H., Yamauchi, S., Tozawa, Y., Ueda, M., Hashimoto, K., Köster, P., Dong, Q., Held, K., Kudla, J., Utsumi, T., Uozumi, N.: N-myristoylation and S-acylation are common modifications of $\mathrm{Ca}^{2+}$-regulated Arabidopsis kinases and are required for activation of the SLAC1 anion channel. New Phytol. 218: 1504-1521, 2018.

Shi, H., Ishitani, M., Kim, C., and Zhu, J.-K.: The Arabidopsis thaliana salt tolerance gene SOS1 encodes a putative $\mathrm{Na}^{+} / \mathrm{H}^{+}$ antiporter. - Proc. nat. Acad. Sci. USA. 97: 6896-6901, 2000.

Sun T., Wang Y., Wang M., Li T.T., Zhou Y., Wang X., Shuya, W.,
He, G, Yang, G.: Identification and comprehensive analyses of the $C B L$ and $C I P K$ gene families in wheat (Triticum aestivem L.). - BMC Plant Biol. 15: 269, 2015.

Thoday-Kennedy, E.L., Jacobs, A.K., Roy, S.J. The role of the CBL-CIPK calcium signaling network in regulating ion transport in response to abiotic stress. - Plant Growth Regul. 76: 3-12, 2015.

Tuteja, N.: Abscisic acid and abiotic stress signaling. - Plant. Signal. Behav. 2: 135-138, 2007.

Villano, C., Esposito, S., Carucci, F., Iorizzo, M, Frusciante, L., Carputo, D., Aversano, R. : High-throughput genotyping in onion reveals structure of genetic diversity and informative SNPs useful for molecular breeding. - Mol. Breed. 39: 5, 2019.

Wang, L., Hu, W., Sun, J., Liang, X., Yang, X., Wei, S., Wang, X., Zhou, Y., Xiao, Q., Yang, G., He, G.: Genome-wide analysis of SnRK gene family in Brachypodium distachyon and functional characterization of BdSnRK2.9. - Plant Sci. 237: 33-45, 2015

Weinl, S., Kudla, J.: The CBL-CIPK $\mathrm{Ca}^{2+}$-decoding signaling network: function and perspectives. - New Phytol. 184: 517528, 2009.

Xi, Y., Liu, J., Dong, C., Cheng, Z.M.: The $C B L$ and $C I P K$ gene family in grapevine (Vitis vinifera): genome-wide analysis and expression profiles in response to various abiotic stresses - Front. Plant Sci 8: 978-993, 2017.

Yang, W., Kong, Z., Omo-Ikerodah, E., Xu, W., Li, Q., Xue, Y. Calcineurin B-like interacting protein kinase OsCIPK23 functions in pollination and drought stress responses in rice (Oryza sativa L.). - J. Genet. Genomics 35: 531-543, 2008.

Yu, Q.Y., An, L.J., Li, W.L.: The CBL-CIPK network mediates different signaling pathways in plants. - Plant Cell Rep. 33: 203-214, 2014.

Yu, Y., Xia, X., Yin, W., Zhang, H.: Comparative genomic analysis of CIPK gene family in Arabidopsis and Populus. Plant Growth Regul. 52: 101-110, 2007.

Zhang, F., Li, L., Jiao, Z., Chen, Y., Liu, H., Chen, X., Fu, J., Wang, G., Zheng, J.: Characterization of the calcineurin B-like $(C B L)$ gene family in maize and functional analysis of ZmCBL9 under abscisic acid and abiotic stress treatments. Plant Sci. 253: 118-129, 2016.

Zhang, H., Yang, B., Liu, W. Z., Li, H., Wang, L., Wang, B., Deng, M., Liang, W., Deyholos, M.K., Jiang, Y.Q.: Identification and characterization of $C B L$ and $C I P K$ gene families in canola (Brassica napus L.). - BMC Plant Biol. 14: 8, 2014.

Zhu, K., Chen, F., Liu, J., Chen, X., Hewezi, T., Cheng, Z. M.: Evolution of an intron-poor cluster of the CIPK gene family and expression in response to drought stress in soybean. - Sci. Rep. 6: 28225, 2016. 